\title{
Purulent Pericarditis Caused by Bacteroides fragilis: A Rare Complication of Cholangitis
}

\author{
Samer Saouma $^{\mathrm{a}}$, Peter C. Olson ${ }^{\mathrm{a}}$, Asif Uddin ${ }^{\mathrm{a}, \mathrm{d}}$, Jonathan Spagnola ${ }^{\mathrm{b}}$, \\ Neville Mobarakaic ${ }^{\mathrm{c}}$ James C. Lafferty ${ }^{\mathrm{b}}$
}

\begin{abstract}
Bacterial infection is a rare cause of pericarditis especially in the post-antibiotic era. When compared to viral or idiopathic etiologies, purulent pericarditis carries a higher risk for complications. While most cases are due to Staphylococcus aureus, we present a rare case of pericarditis due to Bacteroides fragilis originating from a liver abscess and leading to pericardial effusion. Our case highlights the need to maintain a high clinical suspicion of bacterial infection when patients present with sepsis and have evidence of pericarditis.
\end{abstract}

Keywords: Tamponade; Purulent pericarditis; Cholangitis; Liver abscess; Bacteroides

\section{Introduction}

Pericarditis involves inflammation of the pericardium and viral infections are typically the most common cause. However, about $1 \%$ of cases are due to a bacterial infection and carry a poor prognosis if untreated [1]. The etiology of the infection may be hematogenous or extension from a contiguous source. The bacteria identified most commonly are Staphylococcus aureus but can also include Streptococcus pneumoniae, Haemophilus influenza and Neisseria meningitides [2]. Patients can present acutely ill and fever is a common sign. Early diagnosis with pericardial fluid sampling can lead to appropriate therapy and avoid adverse outcomes. We describe a rare case of a patient who presented with pericarditis and pericardial ef-

Manuscript submitted June 15, 2019, accepted August 8, 2019

aDepartment of Medicine, Staten Island University Hospital-Northwell Health, Staten Island, NY, USA

bDepartment of Cardiology, Staten Island University Hospital-Northwell Health, Staten Island, NY, USA

'Department of Infectious Disease, Staten Island University Hospital-Northwell Health, Staten Island, NY, USA

${ }^{\mathrm{d} C o r r e s p o n d i n g ~ A u t h o r: ~ A s i f ~ U d d i n, ~ D e p a r t m e n t ~ o f ~ M e d i c i n e, ~ S t a t e n ~ I s l a n d ~}$ University Hospital-Northwell Health, 500 Seaview Avenue, Suite 225, Staten Island, NY 10305, USA. Email: auddin@northwell.edu

doi: https://doi.org/10.14740/cr904 fusion secondary to a sub-diaphragmatic infection due to $B a c$ teroides fragilis.

\section{Case Report}

An 89-year-old female with a history of cholangitis 4 months prior to arrival presented with anorexia and confusion for 3 days. Her vital signs included a blood pressure of $94 / 56 \mathrm{~mm} \mathrm{Hg}$, a pulse that was irregularly irregular at 144 beats per minute and a temperature of $100.8^{\circ} \mathrm{F}\left(38.2^{\circ} \mathrm{C}\right)$. On physical exam, the patient was toxic-appearing with jugular vein distention and distant heart sounds on auscultation. The patient's clinical condition worsened, and she developed septic shock. Her blood work revealed a leukocyte count of $26,000 / \mu \mathrm{L}$, creatinine of $2.6 \mathrm{mg} /$ $\mathrm{dL}$ and lactic acid of $6.2 \mathrm{mmol} / \mathrm{L}$. Electrocardiogram showed atrial fibrillation with rapid ventricular response. The patient's chest X-ray revealed cardiomegaly. She was intubated and placed on mechanical ventilation. She was started on vasopressor medications and broad-spectrum intravenous antibiotics. An emergent bedside transthoracic echocardiogram showed a large pericardial effusion with evidence of right ventricular collapse. Emergent pericardiocentesis was performed with drainage of $700 \mathrm{~mL}$ of yellowish fluid. Subsequent cultures of the pericardial fluid isolated B. fragilis. Antibiotics were adjusted to ceftriaxone and metronidazole based on the culture sensitivities. After $48 \mathrm{~h}$ of admission, a computerized tomography scan of the abdomen and pelvis with contrast revealed a liver abscess with a fistulous tract reaching the pericardium (Fig. 1). The patient declined drainage of the liver abscess. She received 4 weeks of antibiotic therapy with resolution of her pericarditis and was subsequently discharged to a skilled nursing facility.

\section{Discussion}

Purulent pericarditis is a rare condition with multiple etiologies described in the literature. Historically, pneumonia was cited to be the most common source, but this has significantly decreased in the post-antibiotic era [3]. The organisms associated with pericarditis have also changed over the years. Anaerobic bacteria present a challenge due to the difficulty in isolating organisms as well as its increased resistance to antibiotics. This makes determining the true incidence of infection by anaerobic bacteria difficult. Several reports have documented contigu- 

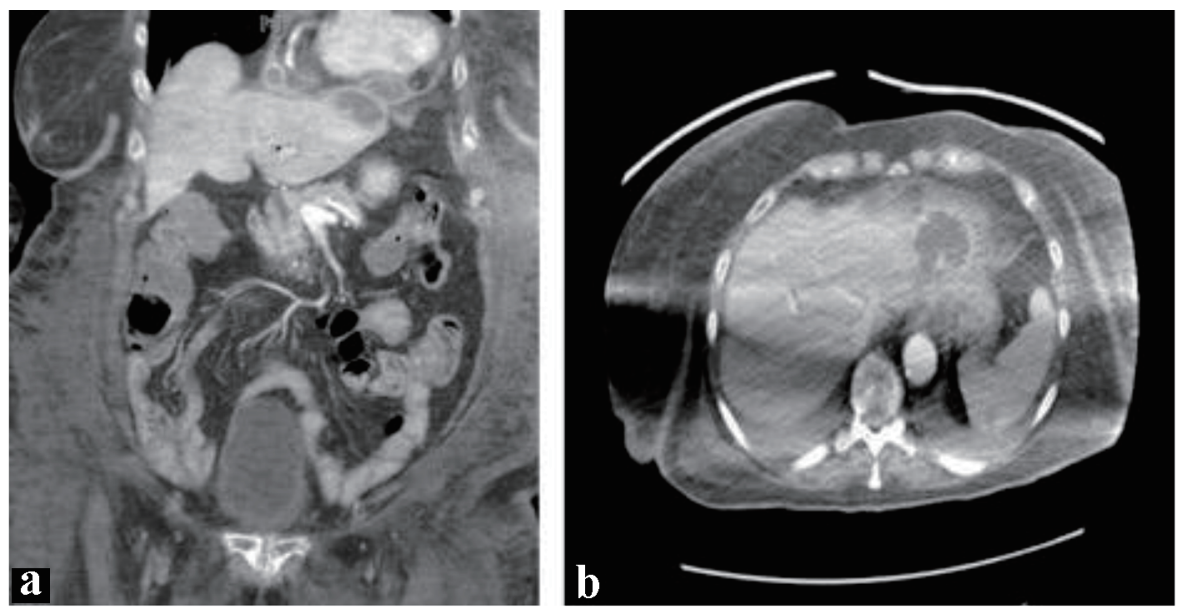

Figure 1. (a) Coronal and (b) axial views of a computed tomography (CT) scan reveal a rim-enhancing lesion in the left lobe of the liver in close contact with a multi-loculated pericardial abscess.

ous or hematogenous spread of anaerobic organisms leading to pericarditis [4]. B. fragilis was one of the common anaerobes isolated in these cases. Bacteroides species are part of the normal flora of the human intestine and cohabits this location with other bacteria without frequent pathogenicity [5]. However, significant infections can occur when the bacterium is displaced outside of the intestines and into the surrounding tissue or bloodstream. An abscess caused by Bacteroides species is prone to rupture leading to bacteremia and systemic infection $[6,7]$.

Our patient likely developed ascending cholangitis due to $B$. fragilis translocation from the intestine to the biliary tract. The cholangitis led to a pyogenic liver abscess which, through a diaphragmatic extension, subsequently caused a purulent pericardial effusion and tamponade. There are few reports of subdiaphragmatic extension from a liver abscess causing purulent pericarditis. The organisms isolated in those cases included bacteria such as Enterococcus faecalis, Escherichia coli, Klebsiella pneumoniae and Staphylococcus epidermidis [8-11]. To our knowledge, this is one of the first reported cases of suspected $B$. fragilis liver abscess causing a purulent pericarditis. Due to the high mortality and variable nature of this condition, we believe that this case offers an opportunity for clinicians to become aware of its existence.

The mainstay of treatment for purulent pericarditis is adequate fluid drainage and tailored antibiotic therapy. Prompt diagnosis and treatment are necessary as cardiac tamponade and constrictive pericarditis are common complications of purulent pericarditis [12]. The mortality rate is estimated to be around $20-30 \%$ [13]. Pericardiocentesis or pericardiotomy is indicated if there is evidence of tamponade or a high clinical suspicion of purulent pericarditis. Intrapericardial thrombolysis is an option in cases where loculated fluid is present or when prevention of constrictive pericarditis is needed [13].

\section{Conclusion}

Purulent pericarditis is a rare diagnosis that carries a high morbidity and mortality if untreated. The clinical presentation can be atypical and definitive diagnosis involves prompt sampling of the pericardial fluid. Aerobic bacteria such as $S$. aureus are commonly isolated but anaerobic bacteria such as $B$. fragilis can also be present and should warrant suspicion of an intra-abdominal source. Antibiotics are the mainstay of therapy and surgical management may be warranted for refractory cases.

\section{Acknowledgments}

None to declare.

\section{Financial Disclosure}

This research did not receive any specific grant from funding agencies in the public, commercial, or not-for-profit sectors.

\section{Conflict of Interest}

The authors declare that there is no conflict of interest.

\section{Informed Consent}

The authors provided and received informed consent from the patient for the writing and reproduction of this case report.

\section{Author Contributions}

JS and SS presented the idea and assisted in the design of the report. SS, PO and AU performed literature review, designed and co-wrote the report. NM and JL provided mentorship and revision of the report. 


\section{References}

1. Adler Y, Charron P, Imazio M, Badano L, Baron-Esquivias G, Bogaert J, Brucato A, et al. 2015 ESC Guidelines for the diagnosis and management of pericardial diseases: the task force for the Diagnosis and Management of Pericardial Diseases of the European Society of Cardiology (ESC) Endorsed by: The European Association for Cardio-Thoracic Surgery (EACTS). Eur Heart J. 2015;36(42):2921-2964.

2. Brook I. Pericarditis caused by anaerobic bacteria. Int J Antimicrob Agents. 2009;33(4):297-300.

3. Sagrista-Sauleda J, Barrabes JA, Permanyer-Miralda G, Soler-Soler J. Purulent pericarditis: review of a 20-year experience in a general hospital. J Am Coll Cardiol. 1993;22(6):1661-1665.

4. Skiest DJ, Steiner D, Werner M, Garner JG. Anaerobic pericarditis: case report and review. Clin Infect Dis. 1994;19(3):435-440.

5. Sears CL, Geis AL, Housseau F. Bacteroides fragilis subverts mucosal biology: from symbiont to colon carcinogenesis. J Clin Invest. 2014;124(10):4166-4172.

6. Brook I. Infective endocarditis caused by anaerobic bacteria. Arch Cardiovasc Dis. 2008;101(10):665-676.

7. Wexler HM. Bacteroides: the good, the bad, and the nitty- gritty. Clin Microbiol Rev. 2007;20(4):593-621.

8. Cho E, Park SW, Jun CH, Shin SS, Park EK, Lee KS, Park SY, et al. A rare case of pericarditis and pleural empyema secondary to transdiaphragmatic extension of pyogenic liver abscess. BMC Infect Dis. 2018;18(1):40.

9. Reddy G, Chatterjee A, Brott BC. Transdiaphragmatic rupture of hepatic abscess producing purulent pericarditis and pericardial tamponade. Circulation. 2015;131(1):e12.

10. Fidalgo Garcia M, Rodriguez Sanjuan JC, Riano Molleda M, Gonzalez Andaluz M, Real Noval H, Gomez Fleitas M. Purulent pericarditis after liver abscess: a case report. Case Rep Med. 2014;2014:735478.

11. Lainez B, Ruiz V, Berjon J, Lezaun R. Purulent pericarditis complicated by cardiac tamponade secondary to a hydatid cyst-associated hepatic abscess. Rev Esp Cardiol. 2009;62(8):948-949.

12. Imazio M, Brucato A, Maestroni S, Cumetti D, Belli R, Trinchero R, Adler Y. Risk of constrictive pericarditis after acute pericarditis. Circulation. 2011;124(11):12701275.

13. Augustin P, Desmard M, Mordant P, Lasocki S, Maury JM, Heming N, Montravers P. Clinical review: intrapericardial fibrinolysis in management of purulent pericarditis. Crit Care. 2011;15(2):220. 